T. MIKAMI

KODAI MATH. J.

17 (1994), 48-68

\title{
LIMIT THEOREMS ON THE EXIT PROBLEMS FOR SMALL RANDOM PERTURBATIONS OF DYNAMICAL SYSTEMS II
}

Dedicated to Professor Nobuyuki Suita on the occasion of his sixtieth birthday

By Toshio Mikami

\begin{abstract}
We consider small random perturbations of dynamical systems $\left\{u^{\varepsilon}(t)\right\}_{0 \leqq t}$ $(0<\varepsilon)$ on $C\left(S^{1} ; R\right)$ when unperturbed dynamical systems $\left\{u^{0}(t)\right\}_{0 \leqq t}$ have the only one asymptotically stable equilibrium point $g_{0}\left(\in C\left(S^{1} ; R\right)\right)$. The objects under consideration are empirical measures which are marginal measures of empirical processes at the exit time $\tau_{D}^{\varepsilon}$ of $\left\{u^{\varepsilon}(t)\right\}_{0 \leqq t}$ from a bounded domain $D\left(\exists g_{0}\right)$ of $C\left(S^{1} ; R\right),\left\{u^{\varepsilon}(t)\right\}_{0 \leqq t \leqq \tau_{D}^{\varepsilon}}$ and $\left\{u^{\varepsilon}\left(\tau_{D}^{\varepsilon} t\right)\right\}_{0 \leqq t \leqq 1}$.
\end{abstract}

\section{Introduction}

Let $S^{1}$ denote the unit circle and consider the following equation;

$$
\begin{gathered}
\partial u_{k}^{\varepsilon}(t, x) / \partial t=D_{k} \partial^{2} u_{k}^{\varepsilon}(t, x) / \partial x^{2}+f_{k}\left(x, u_{1}^{\varepsilon}(t, x), \cdots,\right. \\
\left.u_{d}^{\varepsilon}(t, x)\right)+\varepsilon^{1 / 2} \partial^{2} \zeta_{k}(t, x) / \partial t \partial x, \\
u_{k}^{\varepsilon}(0, x)=g_{k}(x), \quad t>0, \quad x \in S^{1}, \quad k=1, \cdots, d, \varepsilon>0 .
\end{gathered}
$$

Here $D_{k}(k=1, \cdots, d)$ are positive constants, and $f_{k}\left(x, u_{1}, \cdots, u_{d}\right)(k=1, \cdots, d)$ are Lipschitz continuous functions of $S^{1} \times R^{d}$ to $R$, and $\zeta_{k}(t, x)(k=1, \cdots, d)$ are independent Brownian sheets, and $g_{k}(x)(k=1, \cdots, d)$ are continuous functions of $S^{1}$ to $R$ (see Walsh [19]).

It is known that the equation (0.1) has a unique generalized solution $u^{\varepsilon}(t, x)$ $\equiv\left\{u_{k}^{\varepsilon}(t, x)\right\}_{k=1}^{d}$. Moreover $u^{\varepsilon}(t) \equiv u^{\varepsilon}(t, \cdot)$ is a strong Markov process on the space of continuous functions $C\left(S^{1} ; R^{d}\right)$ of $S^{1}$ to $R^{d}$ (see Freidlin [6], Theorem 1) with the sup norm $|\cdot| c(S 1 ; R d)$. By a generalized solution of $(0.1)$, we mean a measurable function $u^{\varepsilon}(t, x)$ such that for any infinitely differentiable function $\varphi$ of $S^{1}$ to $R, t>0$, and $k=1, \cdots, d$,

Received February 18, 1993 ; revised September 17, 1993. 


$$
\begin{aligned}
& \int_{S 1} u_{k}^{\varepsilon}(t, x) \varphi(x) d x-\int_{S^{1}} g_{k}(x) \varphi(x) d x \\
& =\int_{0}^{t} \int_{S^{1}}\left[u_{k}^{\varepsilon}(s, x) D_{k}\left(d^{2} \varphi(x) / d x^{2}\right)+f_{k}\left(x, u^{\varepsilon}(s, x)\right) \varphi(x)\right] d s d x \\
& \quad+\varepsilon^{1 / 2} \int_{S 1} \varphi(x) \zeta_{k}(t, d x),
\end{aligned}
$$

with probability 1 (see Freidlin [6] and Walsh [19]).

The study of the asymptotic behavior of $u^{\varepsilon}(t)$ can be applied to the study of tonnelling (see Faris and Jona-Lasinio [3]) and has connection with the stochastic field theory (see Jona-Lasinio and Mitter [10] and references therein).

Before we state the large deviations theorem for $u^{\varepsilon}(t)$ obtained by Freidlin $[6]$, let us give some notations.

Denote by $W_{2}^{1,2}\left([0, T] \times S^{1} ; R^{d}\right)$ the space of functions of $[0, T] \times S^{1}$ to $R^{d}$ with square integrable first and second order generalized derivatives in $t \in[0, T]$ and in $x \in S^{1}$, respectively. Define the operator $b$ from $W_{2}^{2}\left(S^{1} ; R^{d}\right)$ (三the space of functions of $S^{1}$ to $R^{d}$ with square integrable second order generalized derivatives) to $L^{2}\left(S^{1} ; R^{d}\right)$ (三the space of square integrable functions from $S^{1}$ to $R^{d}$ ) by

$$
b(\varphi)(x) \equiv\left(D_{k}\left(d^{2} \varphi_{k}(x) / d x^{2}\right)+f_{k}(x, \varphi(x))\right)_{k=1}^{d} \quad \text { for } \varphi \in W_{2}^{2}\left(S^{1} ; R^{d}\right) .
$$

Put

$$
S_{0 T}(\varphi)=\left\{\begin{array}{c}
\int_{0}^{T}|d \varphi(t) / d t-b(\varphi(t))|_{L^{2}(S 1 ; R d)}^{2} d t / 2 \\
\quad \text { if } \varphi \in W_{2}^{1,2}\left([0, T] \times S^{1} ; R^{d}\right), \\
\infty \quad \text { if } \varphi \in C\left([0, T] \times S^{1} ; R^{d}\right) \backslash W_{2}^{1,2}\left([0, T] \times S^{1} ; R^{d}\right) .
\end{array}\right.
$$

Then the following result is known.

Theorem 0.1 (Freidlin [6], Theorem 6). For any $T>0, S_{0 T}(\varphi) / \varepsilon$ is the action functional for $\left(P_{g}, u_{t}^{\varepsilon}\right)_{0 \leq t \leq T, g \in C\left(S 1 ; R^{d}\right)}$, as $\varepsilon \rightarrow 0$, in $C\left([0, T] ; C\left(S^{1} ; R^{d}\right)\right)$, that is, the followings hold.

(O) For any $s>0$ and $g \in C\left(S^{1} ; R^{d}\right)$ the following set $\Phi_{g,[0, T]}(s)$ is compact in $C\left([0, T] ; C\left(S^{1} ; R^{d}\right)\right)$ with sup norm;

$$
\Phi_{g[0, T]}(s) \equiv\left\{\varphi \in C\left([0, T] ; C\left(S^{1} ; R^{d}\right)\right) ; \varphi(0)=g, S_{0 T}(\varphi) \leqq s\right\} .
$$

$S_{0 T}(\cdot)$ is lower semicontinuous in $C\left([0, T] \times S^{1} ; R^{d}\right)$.

(I) For any $g \in C\left(S^{1} ; R^{d}\right), s>0$ and open set $O \subset C\left([0, T] ; C\left(S^{1} ; R^{d}\right)\right)$,

$$
-\inf \left\{S_{0 T}(\varphi) ; \varphi(0)=g, \varphi \in O\right\} \leqq \liminf _{\varepsilon \rightarrow 0} \varepsilon \log P_{\boldsymbol{g}}\left(u^{\varepsilon} \in O\right),
$$

uniformly in $g$ and $O$ for which $\inf \left\{S_{0 T}(\varphi) ; \varphi(0)=g, \varphi \in O\right\} \leqq s$. 
(II) For any $g \in C\left(S^{1} ; R^{d}\right), s>0$ and closed set $A \subset C\left([0, T] ; C\left(S^{1} ; R^{d}\right)\right)$,

$$
\limsup _{\varepsilon \rightarrow 0} \varepsilon \log P_{g}\left(u^{\varepsilon} \in A\right) \leqq-\inf \left\{S_{0 T}(\varphi) ; \varphi(0)=g, \varphi \in A\right\},
$$

uniformly in $g$ and $A$ for which $\inf \left\{S_{0 T}(\varphi) ; \varphi(0)=g, \varphi \in A\right\} \leqq s$.

Remark 0.1. From Theorem 0.1 , for any $T>0, \delta>0$ and any $g \in C\left(S^{1} ; R^{d}\right)$,

$$
\lim _{\varepsilon \rightarrow 0} P_{g}\left(\sup _{0 \leqq t \leqq T}\left|u^{s}(t)-u^{0}(t ; g)\right|_{C(S 1 ; R d)}<\delta\right)=1,
$$

where $u^{0}(t ; g)$ denotes the solution to $(0.1)$ with $\varepsilon=0$ and with $u^{0}(0 ; g)=g$.

Let $D$ denote the bounded domain of $C\left(S^{1} ; R^{d}\right)$, with a sufficiently smooth boundary $\partial D$ (see (H.1) below), which contains the only one asymptotically stable equilibrium point $g_{0}$ of $u^{0}$ (see (H.2)). Put

$$
\tau_{C}^{\varepsilon} \equiv \inf \left\{t>0 ; u^{\varepsilon}(t) \notin D\right\} .
$$

In this paper we consider, as $\varepsilon \rightarrow 0$, the asymptotic behavior of $\left\{u^{\varepsilon}\left(\tau_{D}^{\varepsilon} t\right)\right\}_{0 \leqq t \leq 1}$ and that of $\left\{u^{\varepsilon}(t)\right\}_{0 \leqq t \leq \tau_{D}^{\varepsilon}}$, and that of the following empirical measures on $\bar{D} \equiv$ $D \cup \partial D$;

$$
\mu^{\varepsilon}(B) \equiv \int_{0}^{\tau_{D}^{\varepsilon}} 1_{B}\left(u^{\varepsilon}(t)\right) d t / \tau_{D}^{\varepsilon},
$$

where $1_{B}$ denotes the indicator function for Borel set $B \subset C\left(S^{1} ; R^{d}\right)$. The finite dimensional case was considered in Mikami $[14,15]$. Although the results in this paper can be proved for a more general class of stochastic processes, we only consider the process in $(0.1)$ for the sake of simplicity. For a large deviations theorem for a more general class of stochastic processes, see Sowers [18].

Remark 0.2. We only consider the case the equilibrium point $g_{0} \in D$ is of attracting type. In the infinite dimensional case, the equilibrium point can not be repulsive, since the stable manifold is always infinite dimensional (see Foias, Sell and Temam [4], Mallet-Paret and Sell [12] and reference therein). This is a difference between the finite dimensional case and infinite dimensional one. We think that the sets $A_{2} \cup A_{3}$ in Mikami [15] is empty if $\bar{D}=\left\{g_{0}\right\} \cup A_{1}$ $\cup A_{2} \cup A_{3}$, in the infinite dimensional case (see Mikami [15], (H. D)). Proposition 4.2 is a class of example for which $U_{\gamma}\left(g_{0}\right) \backslash\left\{g_{0}\right\} \cup A_{1} \cup A_{2} \cup A_{3}$ is not empty for any $\gamma$ (see Appendix). Here $U_{\gamma}\left(g_{0}\right)$ denotes an $\gamma$-neighborhood of $g_{0}$.

Let us give the assumptions precisely.

(H.1) $D$ is a bounded domain of $C\left(S^{1} ; R^{d}\right)$, with a sufficiently smooth boundary $\partial D$ in the following sense. There exist $\gamma_{0}>\gamma_{1} / 2>0$ such that the followings hold; there exists a continuous function

$$
n: U_{\gamma_{0}}(\partial D) \cap \bar{D} \longmapsto C\left(S^{1} ; R^{d}\right)
$$


for which

$$
\begin{gathered}
g+t n_{g} \in D^{c} \quad \text { if } g \in U_{\gamma_{1} / 2}(\partial D) \cap \bar{D}, 2 \operatorname{dist}(g, \partial D) \leqq t<\gamma_{1}, \\
g-t n_{g} \in D \quad \text { if } 0<t<\gamma_{1}, g \in U_{\gamma_{0}}(\partial D) \cap \bar{D},
\end{gathered}
$$

where we put $U_{\gamma_{0}}(\partial D) \equiv\left\{g \in C\left(S^{1} ; R^{d}\right) ; \operatorname{dist}(g, \partial D)<\gamma_{0}\right\}$, and for which for any $T>0$ and $s>0$, there exists $C(T, s)>0$ such that

$$
S_{0 T}\left(n_{\varphi(\cdot)}\right) \leqq C(T, s)\left(S_{0 T}(\varphi)+1\right),
$$

for all $\varphi$ for which $S_{0 T}(\varphi) \leqq s$.

(H.2) There exists a unique asymptotically stable equilibrium point $g_{0} \in D$ of $u^{0}(t)$ and for any $g \in \bar{D}, u^{0}(t ; g) \in D$ for all $t>0$.

Remark 0.3. (H.1) holds if $D=\left\{g \in C\left(S^{1} ; R^{d}\right) ;|g|_{C(S 1 ; R d)}<1\right\}$ with $n_{g} \equiv g$. (H.2) holds if $f(x, u)=-u$.

In section 1 we state our results which should be compared with the finite dimensional case, Mikami $[14,15]$. In section 2 we give lemmas which will be used in section 3 . In section 3 we prove our results. In section 4 , we consider a class of example which convinces us that $A_{2} \cup A_{3}$ in Mikami [15] is empty if $\bar{D}=\left\{g_{0}\right\} \cup A_{1} \cup A_{2} \cup A_{3}$ in the infinite dimensional case.

\section{Main results}

In this section we state our results.

First of all, we give the large deviations theorem for the empirical measures $\mu^{\varepsilon}$ (see $(0.10)$ ), as $\varepsilon \rightarrow 0$. Before we state the theorem, let us give notations and the definition of the normalized action functional for $\mu^{\varepsilon}$, as $\varepsilon \rightarrow 0$.

Let us denote by $\delta(\bar{D})$ the set of Borel probability measures on $\bar{D}$ with the Prohorov metric $\rho$ (see Ikeda and Watanabe [9], Chap. 1). For a function $\{\varphi(t)\}_{0 \leqq t \leqq T}(T>0)$, put

$$
\mu_{\varphi, T}(d g) \equiv \int_{0}^{T} 1_{\{d g\}}(\varphi(t)) d t / T
$$

(see below (0.10) for notation).

Definition 1.1. For $g \in D$ and $\mu \in \wp(\bar{D})$, put

$$
L_{g}(\mu)= \begin{cases}0 & \text { if } \mu=\delta_{\boldsymbol{g}_{0}}, \\ \inf \left\{S_{0 T}(\varphi) ; \varphi(0)=g, \varphi(T) \in \partial D, \mu_{\varphi, T}=\mu, T<\infty\right\} & \text { otherwise, }\end{cases}
$$

where $\delta_{g_{0}}$ denotes the delta measure on $\left\{g_{0}\right\}$. If the set over which the infimum is taken in (1.2) is empty, then we consider that the infimum is $\infty$.

The following theorem will be proved in section 3 . 
THEOREM 1.1. Suppose that (H.1)-(H.2) hold. Then $L_{g}(\mu) / \varepsilon$ is the action functional for $\mu^{\varepsilon}$ as $\varepsilon \rightarrow 0$, that is, the followings hold.

(O) For any $g \in D$ and any $s>0$, the following set is compact in $\wp(\bar{D})$;

$$
\Phi_{g}(s) \equiv\left\{\mu \in \delta(\bar{D}) ; L_{g}(\mu) \leqq s\right\} .
$$

(I) For any open subset $O$ of $\delta(\bar{D})$ and $g \in D$,

$$
-\inf \left\{L_{g}(\mu) ; \mu \in O\right\} \leqq \liminf _{\varepsilon \rightarrow 0} \varepsilon \log P_{g}\left(\mu^{\varepsilon} \in O\right) .
$$

(II) For any closed subset $A$ of $\delta(\bar{D})$ and $g \in D$,

$$
\limsup _{\varepsilon \rightarrow 0} \varepsilon \log P_{g}\left(\mu^{\varepsilon} \in A\right) \leqq-\inf \left\{L_{g}(\mu) ; \mu \in A\right\} .
$$

The following Corollary 1.1 can be obtained from Theorem 1.1, Freidlin and Wentzell [7], p. 86, Theorem 3.4 by taking $A=\wp(\bar{D})$ and from (1.2).

COROLlaRY 1.1. Suppose that (H.1)-(H.2) hold. Then the following holds; for any $g \in D$ and any $\gamma>0$,

$$
\lim _{\varepsilon \rightarrow 0} P_{g}\left(\rho\left(\mu^{\varepsilon}, \delta_{g_{0}}\right)<\gamma\right)=1 .
$$

Before we state the next result, let us give the following notation; for a function $\{\varphi(t)\}_{0 \leqq t}$, put

$$
T(\varphi) \equiv \inf \{t>0 ; \varphi(t) \notin D\}
$$

THEOREM 1.2. Suppose that (H.1) holds. Then $S_{0 T(\varphi)}(\varphi) / \varepsilon$ is the local action functional with the decay order $\varepsilon^{-1}$ for $\left\{u^{\varepsilon}(t)\right\}_{0 \leq t \leq \tau_{D}^{\varepsilon}}$ as $\varepsilon \rightarrow 0$, that is, for any $g \in D$ and $\varphi \in C([0, \infty) ; \bar{D})$ for which $\varphi(0)=g$ and for which

$$
\inf _{t>0} \operatorname{dist}(\varphi(t), \partial D)=0,
$$

we have the followings;

$$
\begin{aligned}
& \lim _{\gamma \rightarrow 0} \liminf _{\varepsilon \rightarrow 0} \varepsilon \log P_{g}\left(\sup _{0 \leqq t \leq \tau_{D}^{\varepsilon}}\left|u^{\varepsilon}(t)-\varphi(t)\right|_{C(S 1 ; R d}<\gamma\right)=-S_{0 T(\varphi)}(\varphi), \\
& \lim _{\gamma \rightarrow 0} \limsup _{\varepsilon \rightarrow 0} \varepsilon \log P_{g}\left(\sup _{0 \leqq t \leqq \tau_{D}^{\varepsilon}}\left|u^{\varepsilon}(t)-\varphi(t)\right|_{C(S 1 ; R d)}<\gamma\right)=-S_{0 T(\varphi)}(\varphi) .
\end{aligned}
$$

Before we state another result, we give the following definition.

Definition 1.2. For $\varphi \in C([0,1] ; \bar{D})$, put 


$$
S_{01}^{D}(\varphi) \equiv\left\{\begin{array}{cc}
\inf \left\{S_{0 T}(\phi) ; \phi(T t)=\varphi(t), 0 \leqq t \leqq 1, \text { for some } T>0\right\} \\
\left(=|d \varphi / d t|_{L^{2}\left([0, T] \times S 1 ; R^{d}\right)}|b(\varphi)|_{L^{2}\left([0, T] \times S^{1} ; R^{d}\right)}\right. \\
\left.-\langle d \varphi / d t, b(\varphi)\rangle_{L^{2}\left([0, T] \times S 1 ; R^{d}\right)}\right) & \text { if } \varphi(1) \in \partial D, \\
+\infty & \text { otherwise. }
\end{array}\right.
$$

Finally we state the result on $\left\{u^{\varepsilon}\left(\tau_{D}^{\varepsilon} t\right)\right\}_{0 \leqq t \leqq 1}$.

Theorem 1.3. Suppose that (H.1)-(H.2) hold. Then $S_{01}^{D}(\varphi) / \varepsilon$ is the local action functional with the decay order $\varepsilon^{-1}$ for $\left\{u^{\varepsilon}\left(\tau_{D}^{\varepsilon} t\right)\right\}_{0 \leqq t \leqslant 1}$ as $\varepsilon \rightarrow 0$, that $2 s, S_{01}^{D}(\cdot)$ is lower semicontinuous on $C([0,1] ; \bar{D})$; for any $g \in D$ and $\varphi \in C([0,1] ; \bar{D})$ for which $\varphi(0)=g$ and for which $\varphi(1) \in \partial D$, we have the followings;

$$
\begin{aligned}
& \lim _{\gamma \rightarrow 0} \liminf _{\varepsilon \rightarrow 0} \varepsilon \log P_{g}\left(\sup _{0 \leq t \leqq 1}\left|u^{\varepsilon}\left(\tau_{D}^{\varepsilon} t\right)-\varphi(t)\right|_{C(S 1 ; R d)}<\gamma\right)=-S_{01}^{D}(\varphi), \\
& \lim _{\gamma \rightarrow 0} \limsup _{\varepsilon \rightarrow 0} \varepsilon \log P_{g}\left(\sup _{0 \leqq t \leqq 1}\left|u^{\S}\left(\tau_{D}^{\varepsilon} t\right)-\varphi(t)\right|_{C(S 1 ; R d)}<\gamma\right)=-S_{01}^{D}(\varphi) .
\end{aligned}
$$

Remark 1.1. By the lower semicontinuity of $S_{01}^{D}(\cdot)$, we get, from Theorem 1.3, the following;

(I) For any open subset $O$ of $C([0,1] ; \bar{D})$ and $g \in D$,

$$
-\inf \left\{S_{01}^{D}(\varphi) ; \varphi \in O, \varphi(0)=g, \varphi(1) \in \partial D\right\} \leqq \liminf _{\varepsilon \rightarrow 0} \varepsilon \log P_{g}\left(u^{\varepsilon}\left(\tau_{D}^{\varepsilon} \cdot\right) \in O\right),
$$

(II) For any compact subset $K$ of $C([0,1] ; \bar{D})$ and $g \in D$,

$$
-\inf \left\{S_{01}^{D}(\varphi) ; \varphi \in K, \varphi(0)=g, \varphi(1) \in \partial D\right\} \geqq \lim \sup _{\varepsilon \rightarrow 0} \varepsilon \log P_{g}\left(u^{\varepsilon}\left(\tau_{D}^{\varepsilon} \cdot\right) \in K\right) .
$$

(1.15) does not hold for all closed set $K$. Otherwise by the contraction principle, $L_{g}\left(\delta_{g_{0}}\right)=\infty$, which contradicts to Theorem 1.1 .

Theorems 1.2 and 1.3 can be proved, from Lemmas 2.5-2.9 (whose proof should be changed in infinite dimensional case), in the same way as in finite dimensional case Mikami [15]. Therefore we omit the proof of the theorems, but prove the lemmas.

\section{Lemmas}

In this section we state and prove lemmas which will be used in section 3. The assumptions (H.1)-(H.2) can be found in section 0 .

The following lemma was used in Freidlin [6], although it was not clearly stated. For the sake of completeness, we give the proof.

LEMMA 2.0. For any bounded set $A \subset C\left(S^{1} ; R^{d}\right)$, any $T>\gamma>0$ and $s \geqq 0$, the set of functions $\left\{\{\varphi(t)\}_{\gamma \unlhd t \leqq T} ; \varphi \in \cup_{g \in A} \Phi_{g,[0, T]}(s)\right\}$ is pre-compact in $C([\gamma, T]$; 
$\left.C\left(S^{1} ; R^{d}\right)\right)$.

Proof. Put

$$
S_{0 T}^{0}(\phi) \equiv \sum_{k=1}^{d} \int_{0}^{T}\left|d \phi_{k}(t, \cdot) / d t-D_{k} \partial^{2} \phi_{k}(t, \cdot) / \partial x^{2}\right|_{L^{2}(S 1 ; R d)}^{2} d t .
$$

We define

$$
B: C\left([0, \infty) ; C\left(S^{1} ; R^{d}\right)\right) \longmapsto C\left([0, \infty) ; C\left(S^{1} ; R^{d}\right)\right)
$$

by the following; for $\phi \in C\left([0, \infty) ; C\left(S^{1} ; R^{d}\right)\right)$

$$
B \phi \equiv \phi+\omega,
$$

with

$$
\begin{gathered}
\hat{\partial} \omega_{k}(t, x) / \partial t=D_{k} \partial^{2} \omega_{k}(t, x) / \partial x^{2}+f_{k}(x, \phi(t, x)+\omega(t, x)), \\
\omega_{k}(0, x)=0, \quad t>0, \quad x \in S^{1}, k=1, \cdots, d .
\end{gathered}
$$

We first show that $\left\{\left\{B^{-1} \varphi(t)\right\}_{\gamma \leqq t \leqq T} ; \varphi \in \cup_{g \in A} \Phi_{g,[0, T]}(s)\right\}$ is precompact in $C\left([r, T] ; C\left(S^{1} ; R^{d}\right)\right)$. For $\varphi \in \cup_{g \in A} \Phi_{g,[0, T]}(s)$,

$$
\begin{gathered}
S_{0 T}(\varphi)=S_{0 T}^{0}\left(B^{-1} \varphi\right)=S_{0 T}^{0}\left(B^{-1} \varphi(\cdot)-B^{-1} u^{0}(\cdot ; \varphi(0))\right), \\
B^{-1} \varphi(0)-B^{-1} u^{0}(0 ; \varphi(0))=0,
\end{gathered}
$$

since

$$
\begin{gathered}
\partial B^{-1} u_{k}^{0}(t, x) / \partial t=D_{k} \partial^{2} B^{-1} u_{k}^{0}(t, x) / \partial x^{2}, \\
B^{-1} u_{k}^{0}(0, x)=u_{k}^{0}(0, x), \quad t>0, x \in S^{1}, k=1, \cdots, d .
\end{gathered}
$$

From (2.5) and Theorem $0.1, \quad(0), \quad\left\{\left\{B^{-1} \varphi(t)-B^{-1} u^{0}(t ; \varphi(0))\right\}_{0 \leqq t \leqq T} ; \varphi \in\right.$ $\left.\cup_{g \in A} \Phi_{g,[0, T]}(s)\right\}$ is pre-compact in $C\left([0, T] ; C\left(S^{1} ; R^{d}\right)\right)$. It is easy to see that $\left\{\left\{B^{-1} u^{0}(t ; \varphi(0))\right\}_{\gamma \leqq t \leqq T} ; \varphi(0) \in A\right\}$ is pre-compact in $C\left([\gamma, T] ; C\left(S^{1} ; R^{d}\right)\right)$. Hence $\left\{\left\{B^{-1} \varphi(t)\right\}_{\gamma \lesssim t \leqslant T} ; \varphi \in \cup_{g \in A} \Phi_{g,[0, T]}(s)\right\}$ is pre-compact in $C\left([\gamma, T] ; C\left(S^{1} ; R^{d}\right)\right)$.

Next we show that $\left\{\left\{\varphi(t)-B^{-1} \varphi(t)\right\}_{0 \leqq t \leqq T} ; \varphi \in \cup_{g \in A} \Phi_{g,[0, T]}(s)\right\}$ is pre-compact in $C\left([0, T] ; C\left(S^{1} ; R^{d}\right)\right)$.

Let us show that $\left\{\left\{\varphi(t)-B^{-1} \varphi(t)\right\}_{0 \leqq t \leqq T} ; \varphi \in \cup_{g \in A} \Phi_{g,[0, T]}(s)\right\}$ is uniformly bounded in $C\left([0, T] ; C\left(S^{1} ; R^{d}\right)\right)$. For $\omega(t, x) \equiv \varphi(t)-B^{-1} \varphi(t)\left(\varphi \in \cup_{g \in A} \Phi_{g,[0, T]}(s)\right)$, by the Ito formula,

$$
\begin{aligned}
\omega_{k}(t, x)= & \int_{0}^{t} E\left[f _ { k } \left(x+\left(2 D_{k}\right)^{1 / 2} W(s), \omega\left(t-s, x+\left(2 D_{k}\right)^{1 / 2} W(s)\right)\right.\right. \\
& \left.\left.+B^{-1} \varphi\left(t-s, x+\left(2 D_{k}\right)^{1 / 2} W(s)\right)\right)\right] d s
\end{aligned}
$$

for $t>0, x \in S^{1}, k=1, \cdots, d$, where $W(t)$ is a 1-dimensional Wiener process (see Ikeda and Watanabe [9]), and where we extend $\omega(t, x)$ and $f(x, u)$, periodically in $x$, to $[0, \infty) \times R$ and $R \times R$, respectively. From (2.7), by the Gronwall's 
inequality,

$$
\left|\omega_{k}(t, x)\right| \leqq C\left(1+t^{3 / 2}+t\left|B^{-1} \varphi\right|_{C([0, T] ; C(S 1 ; R d))}\right) \exp (C t),
$$

for $t>0, x \in S^{1}, k=1, \cdots, d$, where $C$ denotes a constant which governs the linear growth condition of the Lipschitz function $f$.

Let us show that $\left\{\left\{\varphi(t)-B^{-1} \varphi(t)\right\}_{0 \leqq t \leqq T} ; \varphi \in \cup_{g \in A} \Phi_{g,[0, T]}(s)\right\}$ is equicontinuous in $C\left([0, T] ; C\left(S^{1} ; R^{d}\right)\right)$. For $t, h, \alpha>0, k=1, \cdots, d$ and $x, y \in S^{1}$ for which $|x-y|<\alpha$,

$$
\begin{aligned}
&\left|\omega_{k}(t+h, y)-\omega_{k}(t, x)\right| \\
& \leqq \int_{t}^{t+h} E\left[\mid f_{k}\left(y+\left(2 D_{k}\right)^{1 / 2} W(s), \omega\left(t+h-s, y+\left(2 D_{k}\right)^{1 / 2} W(s)\right)\right.\right. \\
&\left.\left.+B^{-1} \varphi\left(t+h-s, y+\left(2 D_{k}\right)^{1 / 2} W(s)\right)\right) \mid\right] d s \\
&+\int_{0}^{t} E\left[\mid f_{k}\left(y+\left(2 D_{k}\right)^{1 / 2} W(s), \omega\left(t+h-s, y+\left(2 D_{k}\right)^{1 / 2} W(s)\right)\right.\right. \\
&\left.+B^{-1} \varphi\left(t+h-s, y+\left(2 D_{k}\right)^{1 / 2} W(s)\right)\right) \\
&-f_{k}\left(x+\left(2 D_{k}\right)^{1 / 2} W(s), \omega\left(t-s, x+\left(2 D_{k}\right)^{1 / 2} W(s)\right)\right. \\
&\left.\left.+B^{-1} \varphi\left(t-s, x+\left(2 D_{k}\right)^{1 / 2} W(s)\right)\right) \mid\right] d s \\
& \leqq h \sup \left\{\left|f_{k}\left(y_{1}, \omega\left(s, y_{1}\right)+B^{-1} \varphi\left(s, y_{1}\right)\right)\right| ; y_{1} \in S^{1}, 0 \leqq s \leqq t+h\right\} \\
&+C\left(f_{k}\right) t\left\{\alpha+\sup \left\{\mid B^{-1} \varphi\left(s_{1}, y_{1}\right)-B^{-1} \varphi\left(s_{2}, y_{2}\right)\right) \mid ;\right. \\
&\left.\left.\left|y_{1}-y_{2}\right|<\alpha, y_{1}, y_{2} \in S^{1},\left|s_{1}-s_{2}\right| \leqq h, 0 \leqq s_{1}, s_{2} \leqq t+h\right\}\right\} \\
&+C\left(f_{k}\right) \int_{0}^{t} \sup \left\{\mid \omega\left(u+h, y_{1}\right)-\omega\left(u, y_{2}\right)\right) \mid ; \\
&\left.\left|y_{1}-y_{2}\right|<\alpha, y_{1}, y_{2} \in S^{1}\right\} d u,
\end{aligned}
$$

where $C\left(f_{k}\right)$ denotes the Lipschitz constant of $f_{k}$.

By the Gronwall's inequality, the proof is over. $\quad$ Q.E.D.

The following lemma can be proved in the same way as in Freidlin and Wentzell [7], p. 110, Lemma 2.2 (see Freidlin [6], p. 687, lines 10-12).

LEMma 2.1. Suppose that (H.2) holds. Then for any sufficiently small $\gamma>0$, there exists $C_{1}>0$ and $T_{1}>0$ such that for all $T>0$,

$$
S_{0 T}(\varphi)>C_{1}\left(T-T_{1}\right),
$$

for all $\{\varphi(t)\}_{0 \leqq t \leqq T}$ for which $\varphi(t) \in \bar{D} \cap U_{\gamma}\left(g_{0}\right)^{c}(0 \leqq t \leqq T)$ and such that for all $T>0$, 


$$
\limsup _{\varepsilon \rightarrow 0} \varepsilon \log P_{g}\left(u^{\varepsilon}(t) \in \bar{D} \cap U_{\gamma}\left(g_{0}\right)^{c}(0 \leqq t \leqq T)\right) \leqq-C_{1}\left(T-T_{1}\right),
$$

uniformly in $g \in \bar{D} \cap U_{\gamma}\left(g_{0}\right)^{c}$.

The following lemma will be used in Lemma 2.3 .

LEMma 2.2. Suppose that (H.2) holds. Then any $r>0$ and $s>0$, there exists $T>0$ such that for any $\{\varphi(t)\}_{0 \lesssim t<\infty}(\subset \bar{D})$ for which $S_{0 \infty}(\varphi) \leqq s$,

$$
\int_{0}^{\infty} 1_{U_{r}\left(g_{0}\right) c}(\varphi(t)) d t \leqq T
$$

Proof. For $\gamma>0$, take $\alpha>0$ sufficiently small so that if $\left|g-g_{0}\right|_{C(S 1 ; R d)} \leqq \alpha$, then

$$
\sup _{0<t}\left|u^{0}(t ; g)-g_{0}\right|_{C\left(S 1 ; R^{d}\right)}<\gamma / 2,
$$

which is possible from (H.2).

Take $\{\varphi(t)\}_{0 \leqq t<\infty}(\subset \bar{D})$ for which $S_{0 \infty}(\varphi) \leqq s$. Put

$$
\begin{aligned}
t^{n} \equiv \begin{cases}\inf \left\{t>0 ;\left|\varphi(t)-g_{0}\right|_{C\left(S 1 ; R^{d}\right)}<\alpha\right\} & \text { for } n=0, \\
\inf \left\{t>t^{n-1} ;\left|\varphi(t)-g_{0}\right|_{C\left(S_{1} ; R^{d}\right)}<\alpha,\right. & \text { for } n \geqq 1, \\
\left.\sup _{t^{n-1<s<t}}\left|\varphi(s)-g_{0}\right|_{C\left(S^{1} ; R^{d}\right)} \geqq \gamma\right\} & \text { for } n=0,\end{cases} \\
s^{n} \equiv \begin{cases}0 & \text { for } n \geqq 1 . \\
\sup \left\{t^{n}>t ;\left|\varphi(t)-g_{0}\right|_{C\left(S^{1} ; R^{d}\right)}=\alpha\right\} & \end{cases}
\end{aligned}
$$

Then from (2.14),

$$
\begin{array}{ll}
\sup _{t^{n-1} \leqq t \leqq s}\left|\varphi(t)-g_{0}\right|_{C\left(S^{1} ; R^{d}\right)<\gamma} & \text { for } n \geqq 1, \\
\inf _{s^{n} \leqq t \geqq t n}\left|\varphi(t)-g_{0}\right|_{C\left(S^{1} ; R^{d}\right) \geqq \alpha} & \text { for } n \geqq 1 .
\end{array}
$$

From Lemma 2.1 and (2.15), there exists $R=R(s, \alpha)>0$ such that

$$
t^{n}-s^{n} \leqq R \quad \text { for } n \geqq 0,
$$

since $S_{0 \infty}(\varphi) \leqq s$, and there exists a constant $C=C(s, \alpha)>0$ such that

$$
\inf _{n \geqq 1} S_{s n t n}(\varphi) \geqq C,
$$

from Lemma 2.0.

Let us show that (2.12) holds from (2.15)-(2.17). 


$$
\begin{aligned}
\int_{0}^{\infty} 1_{U_{\left.r^{(} g_{0}\right) c}(\varphi(t)) d t} & =\left(\int_{0}^{t 0}+\sum_{n=1}^{\infty} \int_{t^{n-1}}^{t n}\right) 1_{U_{\left.r^{(} g_{0}\right) c}(\varphi(t)) d t} \\
& =\left(\int_{0}^{t 0}+\sum_{n=1}^{\infty} \int_{s^{n}}^{t n}\right) 1_{U_{r}\left(g_{0}\right) c}(\varphi(t)) d t \quad \text { (from (2.15) } \\
& =\left(\int_{0}^{t 0}+\sum_{n=1}^{[s / C]} \int_{s^{n}}^{t n}\right) 1_{U_{r}\left(g_{0}\right) c}(\varphi(t)) d t \quad \text { (from (2.17)) } \\
& \leqq R+(s / C) R \quad \text { (from (2.16)), }
\end{aligned}
$$

where $[s / C]$ denotes the integer part of $s / C$.

Q. E. D.

The following lemma is the statement $(\mathrm{O})$ in Theorem 1.1 in section 1.

Lemma 2.3. Suppose that (H.2) hold. Then for any $g \in D$ and any $s>0$, the set $\Phi_{g}(s)$ is compact in $\&(\bar{D})$ (see (1.3)).

Proof. Take $\left\{\mu^{n}\right\}_{n=1}^{\infty} \subset \Phi_{g}(s)$ for which $\mu^{n} \neq \delta_{g_{0}}$. For each $n \geqq 1$, take

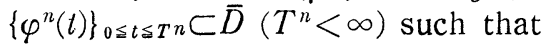

$$
\begin{gathered}
\varphi^{n}(0)=g, \quad \varphi^{n}\left(T^{n}\right) \in \partial D, \quad \mu_{\varphi n, T n}=\mu^{n}, \\
S_{0 T n}\left(\varphi^{n}\right) \leqq s+1 / n .
\end{gathered}
$$

If $\left\{T^{n}\right\}_{n=1}^{\infty}$ is bounded, then there exist a subsequence $\left\{T^{n(k)}\right\}_{k=1}^{\infty}$ of $\left\{T^{n}\right\}_{n=1}^{\infty}$, $T>0$ and a function $\{\varphi(t)\}_{0 \leqq t \leqslant T}$ such that

$$
\begin{gathered}
\lim _{k \rightarrow \infty} T^{n(k)}=T, \quad \varphi(T) \in \partial D, \\
\lim _{k \rightarrow \infty} \sup _{0 \leqq t \leqq \min \left(T, T^{n(k)}\right)}\left|\varphi^{n(k)}(t)-\varphi(t)\right|_{C\left(S^{1} ; R^{d}\right)}=0,
\end{gathered}
$$

(see Mikami [14], (2.11)), and such that

$$
\begin{gathered}
\lim _{k \rightarrow \infty} \mu_{\varphi} n(k), T n(k)=\mu_{\varphi, T}, \\
L_{g}\left(\mu_{\varphi, T}\right) \leqq S_{0 T}(\varphi) \leqq s .
\end{gathered}
$$

This can be proved in the same way as in Mikami [14], Lemma 2.2, (2.11)(2.12) (from Theorem 0.1, (O)).

If $\left\{T^{n}\right\}_{n=1}^{\infty}$ is not bounded, then we can take subsequences $\left\{T^{n(k)}\right\}_{k=1}^{\infty}(\rightarrow \infty)$ and $\left\{\mu^{n(k)}\right\}_{k=1}^{\infty}$ which converges to $\delta_{g_{0}}$, from Lemma 2.2 .

Q. E. D.

The following Lemma 2.4 plays a crucial role in the proof of Theorem 1.1 and can be proved in the same way as in Mikami [15].

LEMma 2.4. Suppose that (H.2) holds. Then for any $g \in D$ and $r>0$, the 
following holds;

$$
\limsup _{T \rightarrow \infty} \limsup _{\varepsilon \rightarrow 0} \varepsilon \log P_{g}\left(\rho\left(\mu^{\varepsilon}, \delta_{g_{0}}\right) \geqq \gamma, \tau_{D}^{\varepsilon} \geqq T\right)=-\infty .
$$

The following lemmas can be proved in the same way as in Mikami [14] (see (2.29)-(2.35) in Lemma 2.7).

Lemma 2.5 (Mikami [14], Lemma 2.5). Suppose that (H.1) holds. Then for any $R>0$ and any $\{\varphi(t)\}_{0 \leqq t \leqq R}$ for which $\varphi(0) \in D, T(\varphi)<R$, and for which $S_{0 R}(\varphi)$ $<\infty$, there exist functions $\left\{\varphi^{n}(t)\right\}_{0 \leqq t \geqq R, 1 \leqq n}$ which exit $\bar{D}$ such that

$$
\begin{gathered}
\varphi^{n}(0)=\varphi(0), \\
T\left(\varphi^{n}\right) \uparrow T(\varphi) \quad \text { as } n \rightarrow \infty, \\
\lim _{n \rightarrow \infty} \sup _{0 \leqq t \leqq R}\left|\varphi^{n}(t)-\varphi(t)\right|_{C\left(S^{1} ; R d\right)}=0, \\
\lim _{n \rightarrow \infty} S_{0 R}\left(\varphi^{n}\right)=S_{0 R}(\varphi) .
\end{gathered}
$$

Proof. In Mikami [14], Lemma 2.5, (2.44), put

$$
\varphi^{k}(t) \equiv\left\{\begin{array}{l}
\varphi(t) \quad \text { for } 0 \leqq t \leqq T(\varphi)-1 / k \text { and } T(\varphi)+1 / k \leqq t \leqq R, \\
\varphi(t)+(t-T(\varphi)+1 / k) n_{\varphi(t)} \quad \text { for } T(\varphi)-1 / k \leqq t \leqq T(\varphi), \\
\varphi(t)+k(T(\varphi)+1 / k-t)(t-T(\varphi)+1 / k) n_{\varphi(t)} \\
\text { for } T(\varphi) \leqq t \leqq T(\varphi)+1 / k .
\end{array}\right.
$$

Then the proof can be acomplished in the same way as in Mikami [14], from (H.1) (see (2.29)-(2.35) in Lemma 2.7).

Q. E. D.

LEMma 2.6 (Mikami [14], Lemma 2.4). Suppose that (H.1) holds. Then for any $T>0$ and any $\{\varphi(t)\}_{0 \leqq t \leqq T}$ for which $\varphi(0) \in D, \varphi(T) \in \partial D, \varphi(t) \in \bar{D}$ for all $0 \leqq$ $t \leqq T$ and for which $S_{0 T}(\varphi)<\infty$, there exist functions $\left\{\varphi^{n}(t)\right\}_{0 \leqq t \leqq T, 1 \leqq n}$ such that

$$
\begin{gathered}
\varphi^{n}(0)=\varphi(0), \quad T\left(\varphi^{n}\right)=T, \\
\lim _{n \rightarrow \infty} \sup _{0 \leq t \leq T}\left|\varphi^{n}(t)-\varphi(t)\right|_{C\left(S^{1} ; R d\right)}=0, \\
\lim _{n \rightarrow \infty} S_{0 T}\left(\varphi^{n}\right)=S_{0 T}(\varphi) .
\end{gathered}
$$

Proof. As in Mikami [14], Lemma 2.4, (2.25), put

$$
\begin{aligned}
& \sigma_{0} \equiv 0, \\
& T_{k} \equiv \inf \left\{t>\sigma_{k-1} ; \varphi(t) \in \partial D\right\},
\end{aligned}
$$




$$
\begin{aligned}
& \tau_{k} \equiv \sup \left\{T_{k}>t ; \operatorname{dist}(\varphi(t), \partial D)>\gamma_{1} / 2\right\}, \\
& \sigma_{k} \equiv \inf \left\{t>T_{k} ; \operatorname{dist}(\varphi(t), \partial D)>\gamma_{1}\right\},
\end{aligned}
$$

for $k \geqq 1$. As in Mikami [14], Lemma 2.4, (2.27), put

$$
\varphi^{m}(t)= \begin{cases}\varphi(t) & \text { if } \sigma_{k-1} \leqq t \leqq \tau_{k}, \\ \varphi(t)-\left(t-\tau_{k}\right) n_{\varphi(t)} / m & \text { if } \tau_{k} \leqq t \leqq \sigma_{k}-1 / m, \\ \varphi(t)-\left(\sigma_{k}-t\right)\left(t-\tau_{k}\right) n_{\varphi(t)} & \text { if } \sigma_{k}-1 / m \leqq t \leqq \sigma_{k} .\end{cases}
$$

Then the proof can be acomplished in the same way as in Mikami [14] (see (2.29)-(2.35) in Lemma 2.7).

Q. E. D.

The following lemma is used to prove Theorem 1.2 whose proof is not given in this paper. Since the proof in finite dimensional case can not be applied to the infinite dimensional case, we give the proof of Lemma 2.7.

LEMMA 2.7. Suppose that (H.1) holds. Then for any function $\{\varphi(t)\}_{0 \leqq t}$ for which $T(\varphi)=\infty, S_{0 \infty}(\varphi) \equiv s(\varphi)<\infty, \inf _{0<t} \operatorname{dist}(\varphi(t), \partial D)=0$,

$$
\begin{gathered}
\lim _{R \rightarrow \infty, \gamma \rightarrow 0} \inf \left\{S_{0 R}(\phi) ; \phi(0)=\varphi(0),\right. \\
\left.\sup _{0 \leqq t \leqq R}|\phi(t)-\varphi(t)|_{C\left(S^{1} ; R d\right)}<\gamma, T(\phi)<R\right\} \\
\leqq S_{0 \infty}(\varphi) .
\end{gathered}
$$

Proof. Take $t_{0}>0$ so that

$$
\sup \left\{|\varphi(t)-\varphi(s)|_{C\left(S^{1} ; R d\right)} ;|t-s|<t_{0}\right\}<\gamma_{1} / 4
$$

(see (H.1) for notation). Take a compact set $K_{1} \subset C\left(S^{1} ; R^{d}\right)$ for which $\left\{\varphi(t)_{0 \leqq t}\right.$ $\subset K_{1}$. This is possible from Lemma 2.0, since $S_{0 \infty}(\varphi)$ is finite. In fact, apply Lemma 2.0 to the set $\left.\{\varphi(k+t) ; 0 \leqq t \leqq 2\}_{k=1}^{\infty} \subset \cup_{g \in D} \Phi_{g,[0,2]}(s(\varphi))\right\}$. Then $\{\varphi(k+t)$; $0 \leqq t \leqq 1\}_{k=2}^{\infty}$ is pre-compact in $C\left([0,1] ; C\left(S^{1} ; R^{d}\right)\right)$. Moreover $\{\varphi(k+t) ; 0 \leqq t \leqq 1\}_{k=1}^{\infty}$ is pre-compact in $C\left([0,1] ; C\left(S^{1} ; R^{d}\right)\right)$ by Theorem $0.1,(\mathrm{O})$.

Put

$$
C_{1} \equiv \max \left(1, \max _{g \in K_{1} \cap U_{\gamma_{1} / 2}(\partial D) \cap \bar{D}}\left|n_{g}\right| C\left(S^{1} ; R d\right)\right),
$$

For any $\gamma\left(\gamma_{1} / 2>\gamma>0\right)$, take $N=N(\gamma)$ such that

$$
2 C_{1} / N(\gamma)<\gamma \text {. }
$$

Put

$$
T^{N} \equiv \inf \{t>0 ; \operatorname{dist}(\varphi(t), \partial D) \leqq 1 / N\},
$$




$$
\varphi^{\gamma}(t) \equiv \begin{cases}\varphi(t) & \left(0 \leqq t \leqq T^{N}-t_{0}\right), \\ \varphi(t)+2 n_{\varphi(t)}\left(t-T^{N}+t_{0}\right) /\left(N t_{0}\right) & \left(T^{N}-t_{0} \leqq t \leqq T^{N}\right) .\end{cases}
$$

Then $\varphi^{r}(0)=\varphi(0)$ and

$$
\sup _{0 \leqq t \leqq T}\left|\varphi^{\gamma}(t)-\varphi(t)\right|<\gamma
$$

from $(2.27)-(2.28)$, and

$$
T\left(\varphi^{\gamma}\right)<T^{N},
$$

since $\varphi^{\gamma}\left(T^{N}\right) \notin D$ from $(0.12)$ and (2.26)-(2.30). Put

$$
C_{0}(\gamma) \equiv 4 \gamma^{2}\left(\pi t_{0}\left(1 / t_{0}+C(f)+\sup _{x \in S^{1},|u| \leqq C_{1}}|f(x, u)|\right)^{2}+S_{\left(T^{N}-t_{0}\right) T^{N}}\left(n_{\varphi}\right)\right) .
$$

Then

$$
S_{0 T^{N}}\left(\varphi^{\gamma}\right) \leqq S_{0 T^{N}}(\varphi)+\left(2 C_{0}(\gamma) S_{\left(T^{N}-t_{0}\right) T^{N}}(\varphi)\right)^{1 / 2}+C_{0}(\gamma) / 2,
$$

since for $t\left(T^{N}-t_{0} \leqq t \leqq T^{N}\right)$,

$$
\begin{aligned}
d \varphi^{\gamma}(t) / d t-b\left(\varphi^{\gamma}(t)\right)= & d \varphi(t) / d t-b(\varphi(t))+f(\cdot, \varphi(t))-f\left(\cdot, \varphi^{\gamma}(t)\right) \\
& +2\left(d n_{\varphi(t)} / d t-b\left(n_{\varphi(t)}\right)\right)\left(t-T^{N}+t_{0}\right) /\left(N t_{0}\right) \\
& +2 f\left(\cdot, n_{\varphi(t)}\right)\left(t-T^{N}+t_{0}\right) /\left(N t_{0}\right)+2 n_{\varphi(t)} /\left(N t_{0}\right) .
\end{aligned}
$$

The right side of (2.34) converges to $S_{0 \infty}(\varphi)$ as $\gamma \rightarrow 0$, from (H.1), which completes the proof.

Q.E.D.

The following lemma will be used to prove Theorem 1.2 and can be proved in the same way as in Mikami [15], Lemma 2.9. Hence we omit the proof.

LEMMA 2.8. For any $g \in D, R>0$ and any $\{\varphi(t)\}_{0 \leq t \leq T} \subset \bar{D}$ for which $\varphi(0)=g$ and for which $T(\varphi) \leqq R$,

$$
\begin{aligned}
& \liminf _{\gamma \rightarrow 0} \inf \left\{S_{0 T}(\phi) ; \phi(0)=g, \phi(T) \in \partial D,\{\phi(t)\}_{0 \leqq t \leqq T} \subset \bar{D},\right. \\
& \sup _{0 \leqq t \leqq T}|\phi(t)-\varphi(t)|_{\left.C\left(S^{1} ; R d\right) \leqq \gamma, T \leqq R\right\}} \\
& \geqq S_{0 T(\varphi)}(\varphi) .
\end{aligned}
$$

The following lemma plays an important role in Remark 1.3 and can be proved in the same way as in Mikami [15].

Lemma 2.9. Suppose that (H.1)-(H.2) hold. Then $S_{01}^{D}(\cdot)$ (see (1.11)) is lower semicontinuous on $C([0,1] ; \bar{D})$. 


\section{Proof of the theorem}

In this section we prove our results. The assumptions (H.1)-(H.2) can be found in section 0 . The proof of Theorem 1.1 is similar to that of Mikami [14]. We give it for the sake of completeness, since $\delta(\bar{D})$ is not compact in the infinite dimensional case.

Proof of theorem 1.1. (O) is proved in Lemma 2.3. We first prove the upper bound (1.5). We devide the proof into the following (3.1)-(3.2); for any closed set $A \subset \wp(\bar{D}), \not \supset \delta_{g_{0}}$ and $g \in D$,

$$
\begin{gathered}
\lim _{R \rightarrow \infty} \limsup _{\varepsilon \rightarrow 0} \varepsilon \log P_{g}\left(\mu^{\varepsilon} \in A, \tau_{D}^{\varepsilon} \leqq R\right) \leqq-\inf \left\{L_{g}(\mu) ; \mu \in A\right\} ; \\
\text { and for any } \gamma>0 \text { and } g \in D, \\
\lim _{R \rightarrow \infty} \limsup _{\varepsilon \rightarrow 0} \varepsilon \log P_{g}\left(\rho\left(\mu^{\varepsilon}, \delta_{g_{0}}\right) \geqq \gamma, \tau_{D}^{\varepsilon} \geqq R\right)=-\infty
\end{gathered}
$$

(see Lemma 2.4). (Notice that the probabilities above are monotone in $R>0$. That is why we can take lim instead of limsup and liminf.)

Before we prove (3.1)-(3.2), let us show that (1.5) holds from (3.1)-(3.2). For $A \subset \xi(\bar{D}), \not \supset \delta_{g 0}$ and $g \in D$,

$$
\begin{aligned}
& \limsup _{\varepsilon \rightarrow 0} \varepsilon \log P_{g}\left(\mu^{\varepsilon} \in A\right) \\
& \leqq \max \left(\lim _{R \rightarrow \infty} \limsup _{\varepsilon \rightarrow 0} \varepsilon \log P_{g}\left(\mu^{\varepsilon} \in A, \tau_{D}^{\varepsilon} \leqq R\right),\right. \\
& \left.\quad \lim _{R \rightarrow \infty} \limsup _{\varepsilon \rightarrow 0} \varepsilon \log P_{g}\left(\rho\left(\mu^{\varepsilon}, \delta_{g_{0}}\right) \geqq \operatorname{dist}\left(A, \delta_{g_{0}}\right), \tau_{D}^{\varepsilon}>R\right)\right) \\
& \leqq-\inf \left\{L_{g}(\mu) ; \mu \in A\right\}
\end{aligned}
$$

from (3.1)-(3.2). For $A \subset \wp(\bar{D}), \exists \delta_{g_{0}}, \gamma>0$ and $g \in D$,

$$
\limsup _{\varepsilon \rightarrow 0} \varepsilon \log P_{g}\left(\mu^{\varepsilon} \in A\right) \leqq 0=-\inf \left\{L_{g}(\mu) ; \mu \in A\right\},
$$

from (1.2), since $A \ni \delta_{g_{0}}$.

Proof of (3.1). For $R>0$, closed set $A \subset \delta(\bar{D}), \not \nexists \delta_{g_{0}}$ and $g \in D$

$$
\begin{aligned}
& \limsup _{\varepsilon \rightarrow 0} \varepsilon \log P_{g}\left(\mu^{\varepsilon} \in A, \tau_{D}^{\varepsilon} \leqq R\right) \\
& \leqq-\inf \left\{S_{0 R}(\varphi) ; \varphi(0)=g, \mu_{\varphi, T} \in A,\right. \\
& \quad \varphi(t) \in \bar{D}(0 \leqq t \leqq T), \varphi(T) \in \partial D, T \leqq R\} \\
& \quad \text { (from Theorem } 0.1 \text {, (II) (see (1.1) for notation)) }
\end{aligned}
$$




$$
\begin{aligned}
=- & \inf \left\{S_{0 T}(\varphi) ; \varphi(0)=g, \mu_{\varphi, T} \in A,\right. \\
& \varphi(t) \in \bar{D}(0 \leqq t \leqq T), \varphi(T) \in \partial D, T \leqq R\} .
\end{aligned}
$$

Let $R \rightarrow \infty$ in (3.5). Then we get (3.1) (see (1.2)).

Q.E. D.

Next we prove the lower bound (1.4). We actually prove the followings; for $\mu \neq \delta_{g_{0}}, g \in D$ and $\gamma>0$,

$$
\lim _{R \rightarrow \infty} \liminf _{\varepsilon \rightarrow 0} \varepsilon \log P_{g}\left(\rho\left(\mu^{\varepsilon}, \mu\right)<\gamma, \tau_{D}^{\varepsilon} \leqq R\right) \geqq-L_{g}(\mu)
$$

; for $g \in D$ and $\gamma>0$,

$$
\liminf _{\varepsilon \rightarrow 0} \varepsilon \log P_{g}\left(\rho\left(\mu^{\varepsilon}, \delta_{g_{0}}\right)<\gamma\right) \geqq 0\left(=-L_{g}\left(\delta_{g_{0}}\right)\right) .
$$

Proof of (3.6). For any $R, \gamma>0, \mu \neq \delta_{g_{0}}$ and $g \in D$,

$$
\begin{aligned}
& \liminf _{\varepsilon \rightarrow 0} \varepsilon \log P_{g}\left(\rho\left(\mu^{\varepsilon}, \mu\right)<\gamma, \tau_{D}^{\varepsilon} \leqq R\right) \\
& \geqq-\inf \left\{S_{0 R}(\varphi) ; \varphi(0)=g, \varphi \in \operatorname{Int}\left\{\phi ; \rho\left(\mu_{\phi, T(\phi)}, \mu\right)<\gamma, T(\phi)<R\right\}\right\} \\
& \text { (from Theorem 0.1, (I) (see (1.1) and (1.7) for notations)) } \\
& =-\inf \left\{S_{0 R}(\varphi) ; \varphi(0)=g, \rho\left(\mu_{\varphi, T(\varphi)}, \mu\right)<\gamma, T(\varphi)<R\right\} \\
& \text { (from Lemma 2.5) } \\
& =-\inf \left\{S_{0 T}(\varphi) ; \varphi(0)=g, \rho\left(\mu_{\varphi, T}, \mu\right)<\gamma, \varphi(t) \in \bar{D}(0 \leqq t \leqq T),\right. \\
& \varphi(T) \in \partial D, T \leqq R\} \quad \text { (from Lemma 2.6) } \\
& \geqq-\inf \left\{S_{0 T}(\varphi) ; \varphi(0)=g, \mu_{\varphi, T}=\mu, \varphi(T) \in \partial D, T \leqq R\right\} .
\end{aligned}
$$

Let $R \rightarrow \infty$ in (3.8). Then we get (3.6).

Q.E. D.

Proof of (3.7). From the upper bound (1.5), for any $g \in D$ and $\gamma>0$,

$$
\lim _{\varepsilon \rightarrow \infty} P_{g}\left(\rho\left(\mu^{\varepsilon}, \delta_{g_{0}}\right) \geqq \gamma\right)=0,
$$

which implies (3.7).

Q.E. D.

\section{Appendix}

In this section we consider the following reaction diffusion equation;

$$
\begin{aligned}
\partial u(t, x ; g) / \partial t & =\left[\partial^{2} u(t, x ; g) / \partial x^{2}\right] / 2+f(u(t, x ; g)) \quad\left(t>0, x \in S^{1}\right), \\
u(0, \cdot ; g) & =g(\cdot)\left(\in C\left(S^{1} ; R\right)\right),
\end{aligned}
$$


where $f(\cdot): R \mapsto R$ is Lipschitz continuous. point.

The following proposition is a fact on the exponentially stable equilibrium

Proposition 4.1. For any stationary solution $g_{0}$ of (4.1) for which

$$
C^{+}\left(g_{0}\right) \equiv \lim \sup _{h \rightarrow 0} \sup _{x \in S^{1}}\left[f\left(h+g_{0}(x)\right)-f\left(g_{0}(x)\right)\right] / h<0,
$$

there exists a constant $c>0$ such that

$$
\sup \left\{\left|u(t ; g)-g_{0}\right|_{C(S 1 ; R)} \exp \left[-C^{+}\left(g_{0}\right) t / 2\right] ; t \geqq 0\right\} \leqq\left|g-g_{0}\right|_{C(S 1 ; R)}
$$

for all $g \in C\left(S^{1} ; R\right)$ for which $\left|g-g_{0}\right|_{C(S 1 ; R)}<c$.

Proof. Take $h>0$ so that

$$
\sup _{x \in S^{1}, 0<|u|<h}\left[f\left(g_{0}(x)+u\right)-f\left(g_{0}(x)\right)\right] / u<C^{+}\left(g_{0}\right) / 2 .
$$

For $g \in C\left(S^{1} ; R\right)$ for which $\left|g-g_{0}\right|_{C(S 1 ; R)}<h$, take $T=T(g, h)>0$ such that

$$
\sup _{0 \leq t \leq T}\left|u(t ; g)-g_{0}\right|_{C(S 1 ; R)}<h,
$$

which is possible from the continuity of $u(t, x: g)$. Then by the Ito formula,

$$
\begin{aligned}
u(t, x ; g)-g_{0}(x) \\
=E\left[\left\{g(x+W(t))-g_{0}(x+W(t))\right\}\right. \\
\quad \times \exp \left(\int _ { 0 } ^ { t } \left[f\left(u(t-s, x+W(s) ; g)-f\left(g_{0}(x+W(s))\right)\right]\right.\right. \\
\left.\left.\quad /\left[u(t-s, x+W(s) ; g)-g_{0}(x+W(s))\right] d s\right)\right]
\end{aligned}
$$

for $0 \leqq t \leqq T$. Here $W(\cdot)$ denotes a 1-dimensional Wiener process (see Ikeda and Watanabe [9]) and we put $[f(a)-f(a)] /[a-a] \equiv d f(a) / d u$. From (4.6),

$$
\left|u(t ; g)-g_{0}\right|_{C(S 1 ; R) \leqq}\left|g-g_{0}\right|_{C(S 1 ; R)} \exp \left[C^{+}\left(g_{0}\right) t / 2\right],
$$

for all $0 \leqq t \leqq T$, which completes the proof from Lemma 2.0 with $s=0$. In fact from Lemma 2.0, $u(t, x ; g)$ is uniformly continuous and $T$ for which (4.7) holds can be taken arbitraly large.

Q.E. D.

If $f(u)=-u / 4$, then from Proposition $4.1,0$ is an exponentially stable equilibrium point. If $f(u)=u / 4$, then 0 is not a stable equilibrium point; $u(t, x ; c)=c \exp (t / 4)$ is a solution for any constant $c$, and by the eigenfunction expansion, 


$$
\lim _{t \rightarrow \infty}|u(t ; g)|_{C(S 1 ; R)}=0 \quad \text { iff } \int_{S 1} g(x) d x=0 .
$$

The following proposition shows that the convergence in (4.8) is not always monotone.

Proposition 4.2. For any stationary solution $g_{0}$ of (4.1) for which

$$
C^{-}\left(g_{0}\right) \equiv \liminf _{h \rightarrow 0} \inf _{x \in S^{1}}\left[f\left(h+g_{0}(x)\right)-f\left(g_{0}(x)\right)\right] / h>0,
$$

and any $\alpha>0$, there exists a function $g$ such that $\int_{S^{1}}\left(g(x)-g_{0}(x)\right) d x=0$, such that $\left|g-g_{0}\right|_{C(S 1 ; R)}<\alpha$ and such that

$$
\sup \left\{\left|u(t ; g)-g_{0}\right|_{C(S 1 ; R)} ; t \geqq 0\right\}>\left|g-g_{0}\right|_{C(S 1 ; R)} .
$$

Proof. Take $h>0$ so that

$$
\inf _{x \in S^{1}, 0<|u|<h}\left[f\left(g_{0}(x)+u\right)-f\left(g_{0}(x)\right)\right] / u>C^{-}\left(g_{0}\right) / 2 .
$$

Take a function $g \in C\left(S^{1} ; R\right)$ such that for $0 \leqq x \leqq 2 \pi$,

$$
g(x)=\left[\exp \left(-R|x-\pi|^{4}\right)-\int_{0}^{2 \pi} \exp \left(-R|y-\pi|^{4}\right) d y /(2 \pi)\right] / R+g_{0}(x) .
$$

Then

$$
\left|g-g_{0}\right|_{C(S 1 ; R)}=g(\pi)-g_{0}(\pi)=[1-C(R)] / R<\alpha,
$$

for sufficiently large $R>0$, where we put

$$
C(R) \equiv \int_{0}^{2 \pi} \exp \left(-R|y-\pi|^{4}\right) d y /(2 \pi) .
$$

For the function $g$ in (4.12), we have, for sufficiently small $t>0$,

$$
\begin{aligned}
& u(t, \pi ; g)-g_{0}(\pi) \\
& \quad \geqq\left|g-g_{0}\right|_{C(S 1 ; R)} \exp \left(-2 R E\left[W(t)^{4}\right] /(1-C(R))+t C^{-}\left(g_{0}\right) / 2\right) \\
& \quad>\left|g-g_{0}\right|_{C(S 1 ; R)} .
\end{aligned}
$$

Let us prove (4.15). By the Ito formula, for $0 \leqq t$,

$$
u(t, \pi ; g)-g_{0}(\pi)
$$




$$
\begin{aligned}
= & E\left[\left\{g(\pi+W(t))-g_{0}(\pi+W(t))\right\}\right. \\
& \times \exp \left(\int_{0}^{t}\left[f(u(t-s, \pi+W(s) ; g))-f\left(g_{0}(\pi+W(s))\right)\right]\right. \\
& \left.\left./\left[u(t-s, \pi+W(s) ; g)-g_{0}(\pi+W(s))\right] d s\right)\right] .
\end{aligned}
$$

Take $T=T(g, h)>0$ as in (4.5). Take $\delta>0$ for which

$$
\exp \left(-R \delta^{4}\right)-C(R)>0
$$

Then there exists $C_{1}=C_{1}(\delta)>0$ such that

$$
\begin{aligned}
u(t, & \pi ; g)-g_{0}(\pi) \\
\geqq & E\left[\left\{\exp \left(-R W(t)^{4}\right)-C(R)\right\} / R\right. \\
& \times \exp \left(\int_{0}^{t}\left[f(u(t-s, \pi+W(s) ; g))-f\left(g_{0}(\pi+W(s))\right)\right]\right. \\
& \left.\left./\left[u(t-s, \pi+W(s) ; g)-g_{0}(\pi+W(s))\right] d s\right) ; \sup _{0 \leqq s \leqq t}|W(s)|<\delta\right] \\
& -C(R) / R \exp (t C(f)) P\left(\sup _{0 \leqq s \geqq t}|W(s)| \geqq \delta\right) \\
\geqq & E\left[\left\{\exp \left(-R W(t)^{4}\right)-C(R)\right\} / R \exp \left(t C^{-}\left(g_{0}\right) / 2\right)\right] \\
& -2 C(R) / R \exp (t C(f)) P\left(\sup _{0 \leqq s \leqq t}|W(s)| \geqq \delta\right) \\
\geqq & E\left[\exp \left(-R W(t)^{4}\right)-C(R)\right] / R \exp \left(t C^{-}\left(g_{0}\right) / 2\right) \\
& -2 C(R) / R \exp (t C(f)) \exp \left(-C_{1} / t\right)
\end{aligned}
$$

for sufficiently small $T(g, h)>t>0$, by the large deviations theory. Here $C(f)$ denotes the Lipschitz constant of $f(u)$, and we considered as follows ;

$$
\begin{aligned}
& P\left(\sup _{0 \leqq s \leqq t}|W(s)| \geqq \delta\right) \\
& \leqq 2 \sup _{0 \leqq s \leqq t} P(|W(s)| \geqq \delta / 2) \text { (see Freidlin and Wentzell [7], p. 151, line 5) } \\
& =2 P\left(t^{1 / 2}|W(1)| \geqq \delta / 2\right)<\exp \left(-C_{1} / t\right),
\end{aligned}
$$

for sufficiently small $t>0$ (see Freidlin and Wentzell [7], Chap. 3, section 2). The last part of (4.18) can be estimated as follows; 


$$
\begin{aligned}
E & {\left[\exp \left(-R W(t)^{4}\right)-C(R)\right] / R \exp \left(t C^{-}\left(g_{0}\right) / 2\right) } \\
& -2 C(R) / R \exp (t C(f)) \exp \left(-C_{1} / t\right) \\
= & \left|g-g_{0}\right| C(S 1 ; R) \exp \left(t C^{-}\left(g_{0}\right) / 2\right)\left\{[ 1 - C ( R ) ] ^ { - 1 } \left(E\left[\exp \left(-R W(t)^{4}\right)-C(R)\right]\right.\right. \\
& \left.\left.-2 C(R) \exp \left(t C(f)-t C^{-}\left(g_{0}\right) / 2-C_{1} / t\right)\right)\right\},
\end{aligned}
$$

and

$$
\begin{aligned}
& {[1-C(R)]^{-1}\left\{E\left[\exp \left(-R W(t)^{4}\right)-C(R)\right]\right.} \\
& \left.\quad-2 C(R) \exp \left(t C(f)-t C^{-}\left(g_{0}\right) / 2-C_{1} / t\right)\right\} \\
& \geqq[1-C(R)]^{-1}\left\{E\left[\exp \left(-R W(t)^{4}\right)-C(R)\right]-\exp \left(-C_{1} /(2 t)\right)\right\}
\end{aligned}
$$

(for sufficiently small $t>0$ )

$$
\begin{aligned}
& =1-\left(1+\exp \left(-C_{1} /(2 t)\right)-E\left[\exp \left(-R W(t)^{4}\right)\right]\right) /[1-C(R)] \\
& \geqq \exp \left(-(3 / 2)\left(1+\exp \left(-C_{1} /(2 t)\right)-E\left[\exp \left(-R W(t)^{4}\right)\right]\right) /[1-C(R)]\right) \\
& \geqq \exp \left(-(3 / 2)\left(\exp \left(-C_{1} /(2 t)\right)+R E\left[W(t)^{4}\right]\right) /[1-C(R)]\right) \\
& \geqq \exp \left(-2 R E\left[W(t)^{4}\right] /[1-C(R)]\right)
\end{aligned}
$$

for sufficiently small $t>0$. In the second and the third inequality, we used the following, respectively ;

$$
\begin{gathered}
1-x \geqq \exp (-3 x / 2) \quad \text { for sufficiently small } x>0, \\
1-\exp (-x) \leqq x, \quad \text { for } x>0 .
\end{gathered}
$$

Q.E.D.

From Proposition 4.2, the behavior of $u(t ; g)$ on $C\left(S^{1} ; R\right)$ and that on $L^{2}\left(S^{1} ; R\right)$ are not always the same for small $t>0$. By the following proposition, they are similar to each other for sufficiently large $t>0$.

PROPOSITION 4.3. For any $s, t>0$, any $g \in C\left(S^{1} ; R\right)$, and any stationary solution $g_{0}$ of $(4.1)$

$$
\begin{aligned}
& \left|u(t ; g)-g_{0}\right|_{L^{2}(S 1 ; R)} \leqq(2 \pi)^{1 / 2}\left|u(t ; g)-g_{0}\right|_{C(S 1 ; R)} \\
& \left|u(t+s ; g)-g_{0}\right|_{C(S 1 ; R)} \exp (-C(f) s) \\
& \quad \leqq(2 /(\pi s))^{1 / 4}\left(1-\exp \left(-2 \pi^{2} / s\right)\right)^{-1 / 2}\left|u(t ; g)-g_{0}\right|_{L 2(S 1 ; R)} .
\end{aligned}
$$
and $t$,

Proof. (4.24) is trivial. Let us prove (4.25). By the Ito formula, for $0<s$ 


$$
\begin{aligned}
\left|u(t+s, x ; g)-g_{0}(x)\right| & \mid E\left[\left\{g(x+W(t+s))-g_{0}(x+W(t+s))\right\}\right. \\
& \times \exp \left(\int_{0}^{t+s}\left[f(u(t+s-a, x+W(a) ; g))-f\left(g_{0}(x+W(a))\right)\right]\right. \\
& \left.\left./\left[u(t+s-a, x+W(a) ; g)-g_{0}(x+W(a))\right] d a\right)\right] \mid \\
= & \mid E\left[\operatorname { e x p } \left(\int_{0}^{s}\left[f(u(t+s-a, x+W(a) ; g))-f\left(g_{0}(x+W(a))\right)\right]\right.\right. \\
& \left./\left[u(t+s-a, x+W(a) ; g)-g_{0}(x+W(a))\right] d a\right) \\
& \left.\times\left\{u(t, x+W(s) ; g)-g_{0}(x+W(s))\right\}\right] \mid \\
& (\text { from the Markov property of } W(\cdot)) \\
\leqq & \exp (C(f) s) E\left[\left\{u(t, x+W(s) ; g)-g_{0}(x+W(s))\right\}^{2}\right]^{1 / 2} .
\end{aligned}
$$

The last part of (4.26) can be estimated as follows;

$$
\begin{aligned}
& E\left[\left\{u(t, x+W(s) ; g)-g_{0}(x+W(s))\right\}^{2}\right] \\
& \quad=\int_{R}\left\{u(t, x+y ; g)-g_{0}(x+y)\right\}^{2} \exp \left(-y^{2} /(2 s)\right)(2 \pi s)^{-1 / 2} d y \\
& \quad=\sum_{k=-\infty}^{\infty} \int_{2 \pi k}^{2 \pi(k+1)}\left\{u(t, x+y ; g)-g_{0}(x+y)\right\}^{2} \exp \left(-y^{2} /(2 s)\right)(2 \pi s)^{-1 / 2} d y,
\end{aligned}
$$

and for any integer $k$,

$$
\begin{aligned}
& \int_{2 \pi k}^{2 \pi(k+1)}\left\{u(t, x+y ; g)-g_{0}(x+y)\right\}^{2} \exp \left(-y^{2} /(2 s)\right)(2 \pi s)^{-1 / 2} d y \\
& \leqq \exp \left(-(2 \min (|k|,|k+1|) \pi)^{2} /(2 s)\right)(2 \pi s)^{-1 / 2}\left|u(t ; g)-g_{0}\right|_{L^{2}(S 1 ; R)}^{2},
\end{aligned}
$$

which completes the proof.

Q.E. D.

Acknowledgement. The author would like to thank Professor H. Matano for the useful discussion on the infinite dimensional dynamical systems. He would also like to thank the referee for making this paper more readable.

\section{REFERENCES}

[1] Brassesco, S., Some results on small random perturbations of an infinite dimensional dynamical system, Stoc. Proc. Appl., 38 (1991), 33-53.

[2] Chow, P. L., Large deviation problem for some parabolic Ito equations, Comm. Pure Appl. Math., 45 (1992), 97-120. 
[3] Faris, W. G. and Jona-Lasinio, G., Large fluctuations for a nonlinear heat equation with noise, J. Phys. A (Math. Gen.), 15 (1982), 3025-3055.

[4] Foias, C., Sell, G. AND Temam, R., Inertial manifolds for nonlinear evolutionary equations, J. Diff. Equa., 73 (1988), 309-353.

[5] Freidlin, M. I., Functional Integration and Partial Differential Equations, Princeton University Press, Princeton, 1985.

[6] Freidlin, M.I., Random perturbations of reaction-diffusion equations . the quasideterministic approximation, Trans. Amer. Math. Soc., 305 (1988), 665-697.

[7] Freidlin, M. I. And Wentzell, A. D., Random Perturbations of Dynamical Systems, Springer-Verlag, Berlin-Heidelberg-New York-Tokyo, 1984.

[8] Galves, A., Olivieri, E. ANd Vares, M.E., Metastability for a class of dynamical systems subject to small random perturbations, Ann. Probab., 15 (1987), $1288-1305$.

[9] IKeda, N. And Watanabe, S., Stochastic Differential Equations and Diffusion Processes, North-Holland/Kodansha, Amsterdam-New York-Oxford-Tokyo, 1981.

[10] Jona-Lasinio, G. And Mitter, P. K., Large deviation estimates in the stochastic quantization of $\varphi_{2}^{4}$, Commun. Math. Phys., 130 (1990), 111-121.

[11] Kifer, Y., Random Perturbations of Dynamical Systems, Birkhäuser, BostonBasel, 1988.

[12] Mallet-Paret, J. ANd Sell, G., Inertial manifolds for reaction diffusion equations in higher space dimensions, J. Amer. Math. Soc., 1 (1988), 805-866.

[13] Martinelli, F., Olivieri, E. And Scoppola, E., Small random perturbations of finite- and infinite-dimensional dynamical systems : unpredictability of exit times, J. Stat. Phys., 55 (1989), 477-522.

[14] Miкамi, T., Large deviations theorems for empirical measures in FreidlinWentzell exit problems, Ann. Probab., 19 (1991), 58-82.

[15] Mikami, T., Limit theorems on the exit problems for small random perturbations of dynamical systems I, to appear in Stoc. and Stoc. Rep..

[16] Molchanov, S. A., Tutubalin, V. N. and Wentzell, A. D., Asymptotic problems in probability theory and the theory of random media, Theory Probab. Appl., 35 (1991), 87-93.

[17] Smoller, J., Shock Waves and Reaction-Diffusion Equations, Springer-Verlag, Berlin-Heidelberg-New York-Tokyo, 1983.

[18] Sowers, R.B., Large deviations for a reaction-diffusion equation with nonGaussian perturbations, Ann. Probab., 20 (1992), 504-537.

[19] Walsh, J. B., An introduction to stochastic partial differential equations, École d'Été de Probabilités de Saint Flour XIV-1984 (P. L. Hennequin, ed.), Lect. Notes in Math., 1180, 266-439, Springer-Verlag, Berlin-Heidelberg-New YorkTokyo, 1986.

[20] Wentzell, A. D., Limit Theorems on Large Deviations for Markov Stochastic Processes, Kluwer Academic Publishers, Dordrecht-Boston-London, 1990.

[21] Wentzell, A. D. And Freidlin, M. I., On small random perturbations of dynamical systems, Russian Math. Survey, 25 (1970), 1-55.

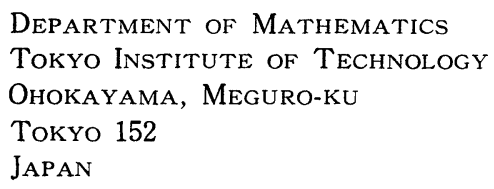

